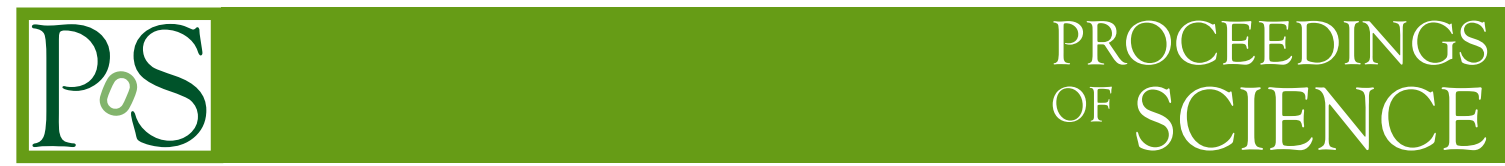

\title{
Restoring jet correlations in high pile-up data
}

\author{
Hans Van Haevermaet* \\ University of Antwerp (BE) \\ E-mail: hans.van.haevermaetecern.ch
}

\begin{abstract}
Experiments in the high-luminosity runs at the Large Hadron Collider face the challenges of very large pile-up. Primary techniques to deal with this are based on precise vertex and track reconstruction. Outside tracker acceptances, however, lie regions of interest for many aspects of the LHC physics program. We explore complementary approaches to pile-up treatment and propose a data-driven jet-mixing method which can be used outside tracker acceptances without depending on Monte Carlo generators.
\end{abstract}

38th International Conference on High Energy Physics 3-10 August 2016

Chicago, USA

${ }^{*}$ Speaker. 


\section{Introduction}

Experiments at hadron colliders operating at a very high luminosity face the challenge of high pile-up conditions, which create a high probability that the final state of a particular process drowns in pile-up activity. Advanced vertex techniques have been developed to deal with this inside regions covered by tracking detectors, but outside, experiments rely on Monte Carlo simulations to model pile-up effects. This, however, introduces a significant model dependence, especially in more forward regions where currently less precise measurements are available to constrain Monte Carlo models.

We propose a novel data-driven approach with a main focus to deal with the presence of high$p_{\mathrm{T}}$ jets produced from pile-up events, in a region where tracking detectors are not available to identify them. An example would be Higgs production through vector boson fusion, with associated jets produced outside tracking detector acceptances, e.g. $|\eta|>3$. This is a different issue than what most of the existing pile-up correction methods treat, which are the jet $p_{\mathrm{T}}$ pedestal, due to the bias in the jet $p_{\mathrm{T}}$ from added pile-up particles in the jet cone, and the clustering of overlapping soft particles from multiple collisions into jets.

We will therefore use an existing method to remove the contribution of soft pile-up particles in the event, and introduce a new approach to treat the misidentification of high- $p_{\mathrm{T}}$ jets in regions where no track and vertex information is available [1]. The aim is to look for a method that can restore correlations between final state particles or jets, can be used without track information, and without a Monte Carlo model. To achieve this we propose to use a minimum bias data sample recorded during high pile-up runs, and apply an event-mixing technique with this sample, to retrieve the true signal from the measured one in high pile-up. One of the features of the method is that it does not require any dedicated data-taking at low pile-up conditions, since the required data can be recorded at the same time as the signal events at high pile-up, so there is no loss in luminosity. We will illustrate this using Drell-Yan with associated jet production as a case study, and discuss the main consequences of additional pile-up collisions.

\section{Drell-Yan with associated jet production at high pile-up}

Consider the production of a Drell-Yan lepton pair via $Z$-boson exchange $\left(60 \mathrm{GeV}<m^{\text {(boson) }}<\right.$ $120 \mathrm{GeV},\left|\eta^{\text {(boson) }}\right|<2$ ) with an associated jet ( $p_{\mathrm{T}}^{\text {(jet) }}>30 \mathrm{GeV},\left|\eta^{\text {(jet) }}\right|<4.5$ ), at a centre-of-mass energy of $\sqrt{s}=13 \mathrm{TeV}$. Figure 1 shows the leading jet $p_{\mathrm{T}}$ (left) and Drell-Yan $p_{\mathrm{T}}$ (right) spectra with and without pile-up. In addition a curve is present that shows the result of applying a SoftKiller (SK) [2] correction to the pile-up distributions. This method can correct quite well for the presence of a large amount of soft pile-up particles in the event, which leads to a bias (jet pedestal) in jet $p_{\mathrm{T}}$ due to added pile-up particles in the jet cone or can make fake jets when these soft particles are clustered together; but it is clear that it can not treat the contribution from genuine high- $p_{\mathrm{T}}$ jets that come from independent pile-up events.

\section{Uncorrelated event samples and the jet-mixing method}

To treat this remaining contribution we propose to apply jet mixing techniques $[3,4,5,6]$ using uncorrelated samples. The main idea is that the signal at high pile-up is obtained by mixing 

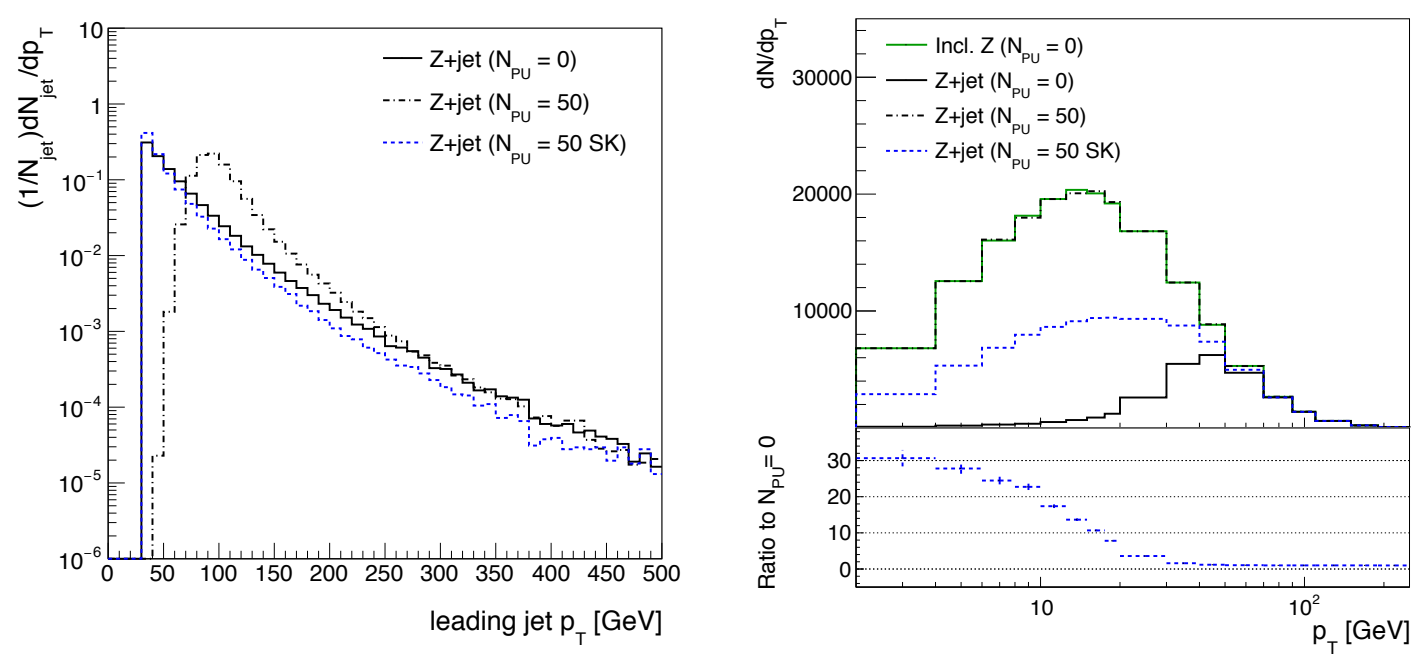

Figure 1: Application of SoftKiller to $Z+$ jet production for the leading jet $p_{\mathrm{T}}$ (left), and $Z$-boson $p_{\mathrm{T}}$ (right) spectra. [1]

the signal without pile-up with a minimum bias sample of data at high pile-up. Thus to identify the contribution of high- $p_{\mathrm{T}}$ jets coming from independent pile-up events, we construct a "signal + pile-up" scenario in a data-driven way, where physics objects from pile-up background are added to the event before selection criteria are applied. The technique is designed to treat the region of high $\mathrm{N}_{\mathrm{PU}}$, where $\left(\mathrm{N}_{\mathrm{PU}}+1\right) / \mathrm{N}_{\mathrm{PU}} \approx 1$. We use the Drell-Yan + jets example to illustrate the method by taking a sample (Monte Carlo in this exercise) containing $\mathrm{N}_{\mathrm{PU}}$ minimum bias events, apply a SoftKiller subtraction to it, and mix it with the signal at zero pile-up, to then perform the same event selection requiring a jet with $p_{T}^{(\text {jet })}>30 \mathrm{GeV}$ and $\left|\eta^{(\text {jet })}\right|<4.5$.
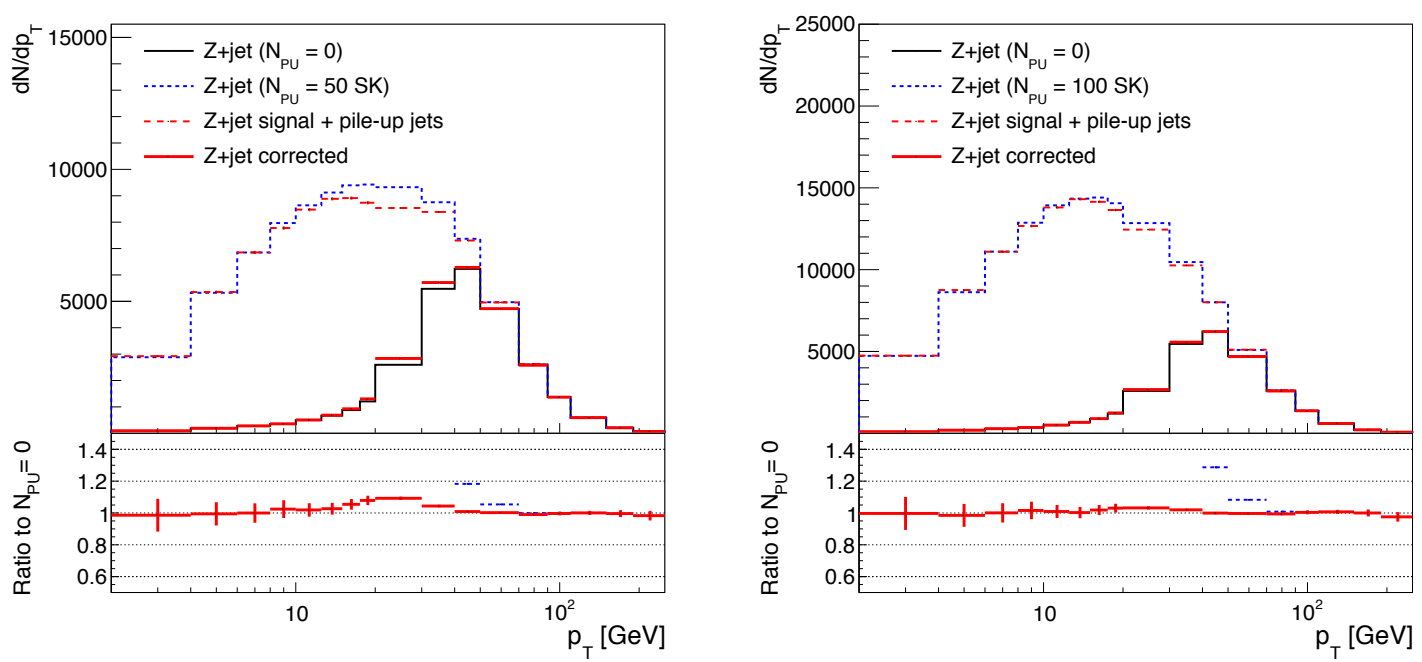

Figure 2: The $Z$-boson $p_{\mathrm{T}}$ spectrum in $Z+$ jet production corrected with the jet-mixing method. Left: $\mathrm{N}_{\mathrm{PU}}=$ 50; right: $\mathrm{N}_{\mathrm{PU}}=100 .[1]$ 
The results are presented in Fig. 2 for $\mathrm{N}_{\mathrm{PU}}=50$ and $\mathrm{N}_{\mathrm{PU}}=100$. The solid black curve is the "true" $Z+$ jet signal, the dashed blue curve is the high pile-up SoftKiller-corrected result, and the solid red curve is the high pile-up SoftKiller + jet-mixing corrected result. We regard the dashed blue curve as pseudodata in high pile-up, and the long-dashed red curve is the jet-mixed curve, obtained as described above. The result of the mixing method (solid red curve) is then obtained by an unfolding, here defined by multiplying the signal at zero pile-up by the ratio of the pseudodata curve to the jet-mixed curve. Without using any Monte Carlo method, the true signal is extracted nearly perfectly from the jet-mixed sample. In addition to the closure test shown above, we have checked the model dependence by applying the jet-mixing technique to different starting distributions, and checked the performance of the jet-mixing method as function of $\mathrm{N}_{\mathrm{PU}}$.

\section{Conclusions}

We proposed a new data-driven approach to treat pile-up, which is model independent, and is able to restore final state correlations without the use of track or vertex information. In particular it is designed to correct for the misidentification of high- $p_{\mathrm{T}}$ jets from independent pile-up events, a contribution that is not treated in existing methods outside tracking detector regions. The main ingredient is a minimum bias data sample recorded during high pile-up runs, which is used to construct a jet-mixing method. Using $Z+$ jet processes as example, we demonstrated that one can successfully extract true signal distributions from the mixed sample, removing any model dependence implied by the use of Monte Carlo event generators. It is a general technique that can be used in any measurement to restore correlations between final-state particles, and does not require special runs at low pile-up. The method thus implies good prospects both for precision Standard Model studies, and for searches for beyond Standard Model physics in high pile-up regimes.

\section{Acknowledgements}

The results in these proceedings have been obtained in collaboration with F. Hautmann and H. Jung. Many thanks to the organisers of the ICHEP 2016 conference for giving us the opportunity to present these studies.

\section{References}

[1] F. Hautmann, H. Jung and H. Van Haevermaet, Phys. Lett. B 754 (2016) 260.

[2] M. Cacciari, G. P. Salam and G. Soyez, Eur. Phys. J. C 75 (2015) 59.

[3] D. Drijard, H. G. Fischer and T. Nakada, Nucl. Instrum. Meth. A 225 (1984) 367.

[4] S. Schael et al. [ALEPH Collaboration], Phys. Lett. B 606 (2005) 265.

[5] H. Schettler, DESY-THESIS-2013-036, CERN-THESIS-2013-265.

[6] B. Dutta, T. Kamon, N. Kolev and A. Krislock, Phys. Lett. B 703 (2011) 475. 